\title{
HYDROPHILIC NANOFIBER MEMBRANES FOR OILY WASTEWATER TREATMENT
}

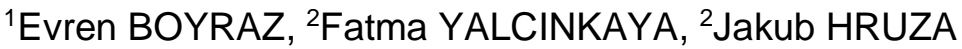 \\ ${ }^{1}$ Faculty of Mechatronics, Technical University of Liberec, Liberec, Czech Republic, EU, evren.boyraz@tul.cz \\ ${ }^{2}$ Institute for Nanomaterials, Advanced Technology and Innovation, Technical University of Liberec, Liberec, \\ Czech Republic, EU, fatma.yalcinkaya@tul.cz
}

https://doi.org/10.37904/nanocon.2020.3703

\begin{abstract}
Electrospun nanofiber seems interesting in the filter domain area due to its highly porous structure, narrow pore size, and high specific surface area. In this work, polyamide 6 (PA6), polyvinylidene fluoride (PVDF), and polyacrylonitrile (PAN) nanofibers were prepared as hybrid membranes. Hydrophobic PVDF and PAN nanofiber membrane surface was hydrophilized using a chemical modification method and performance for the treatment of oily wastewater was compared. The surface modification of the membrane improved membrane anti-fouling property. Results indicated that nanofibrous membranes may become interesting materials for the treatment of oily wastewater.
\end{abstract}

Keywords: Nanofiber, membrane, wastewater, oil separation, hydrophilic

\section{INTRODUCTION}

A large amount of oily wastewater has been generated from various industries such as automotive, mining, food and beverages, pharmaceutical, petrochemical, metallurgical, oil and natural gas exploration, and processing. The release of oily wastewater into nature cause of severe damage to the environment and human health. Oily wastewater with a droplet size less than $10 \mu \mathrm{m}$ is in a highly stable state and very difficult to separate. For this reason, it has to be properly treated before discharge or recycling. There are several techniques to treat oil-water emulsion namely electrostatic coalesce, heat treatment, chemical emulsification, centrifugal settling, and membrane filtration [1]. Some of these methods have certain limitations such as using toxic compounds, high cost, low separation efficiency, demand large space, and generation of secondary pollutants.

Recently, membrane technology has gained huge interest as an effective separation process for the emulsified oil droplets that are smaller than $\sim 10 \mu \mathrm{m}$. Membrane separation offers, high selectivity, easy to operate, costeffectiveness, low energy demand, and no secondary pollution. However, membranes have suffered from fouling due to the deposition of foulant (such as particles, oil droplets, aggregates) on the surface or in the pores of the membranes causing a decline in flux. As a result, the membrane life-span is shortened, and a frequently cleaning process is needed which increases energy demanded and secondary pollution. To overcome membrane fouling, several attempts have been done. These attempts include physical and chemical modification of the membrane surface or blending with other polymers or incorporating additives into the membrane. Zhao et al. [2] grafted perfluoroalkyl groups onto polyacrylonitrile (PAN) membrane surface through the acylation reaction. The fluorinated PAN membranes displayed desirable fouling release property with almost complete flux recovery for oil/water emulsion (separation of oil) due to non-polar hydrophobic perfluoroalkyl groups which reduced the surface free energy and increased the degree of chemical heterogeneity. Similarly, Xu et al.[3] prepared (separation of oil) modified membranes which were highly water repellent, oleophilic, and showed excellent self-cleaning properties. There are plenty of attempts that have 
been reported for the separation of oil-in-water or water-in-oil $[4,5]$. Yet, there is a demand to improve highly permeable and fouling resistance membrane material for the separation of oily wastewater.

Nanofiber membranes offer an effective separation process with high permeability due to their tight pore size, and porous structure. The main advantages of nanofibers in membrane technology are their specific surface area, tight pore size, narrow pore size distribution, and highly porous structure (over $80 \%$ ). The tight pore size and narrow pore size distribution can capture foulants in micron size on their surface that improves the filtration efficiency. A highly porous structure increases membrane flux and permeability. The high specific surface area serves many micropores in the fiber network which improves filtration efficiency and performance. More interestingly, nanofiber that has highly specific surface area can be modified by surface-active chemical compounds to improve their functionality.

In the present work, polyamide 6 (PA6), polyvinylidene fluoride (PVDF), and PAN nanofiber membranes were prepared by using the lamination method, and hydrophobic membranes were modified to be superhydrophilic for separation of oil-water emulsion. PVDF and PAN nanofibers have low wettability compared to PA6. PA6 membranes are swelling in alkaline conditions, due to the dissociation of carboxylic groups in polymer chains which yields in chains to be charged negatively. As a result average pore size of the membrane increases. For the reasons above, only PVDF and PAN membranes have been modified using an alkaline treatment. The nanofiber membranes were modified with no complex preparation method or expensive equipment. Modified membranes showed good permeability and fouling resistance regardless of their original properties. Prepared nanofiber membranes are promising for the practical application of the oil-water separation system.

\section{EXPERIMENTAL}

The polymers PA6, PVDF, and PAN have been prepared by Nanospider electrospinning device at the Technical University of Liberec. The lamination process has been done using the heat-press method onto a hydrophobic nonwoven material. All the nanofibers had an area weight $3 \mathrm{gsm}$. The surface modification for PVDF and PAN membranes have been done using an alkaline solution prepared as $2 \mathrm{~g}$ of potassium hydroxide $(\mathrm{KOH})$ dissolved in $20 \mathrm{~mL}$ of isopropyl alcohol (Penta s.r.o.). Samples were kept in the alkaline solution for $1 \mathrm{~h}$, then rinsed several times and kept in the distilled water. The treated samples are called PVDF-OH and PAN$\mathrm{OH}$. The water contact angle of the membranes has been measured by Krüss Drop Shape Analyzer DS4 (Krüss GmbH, Hamburg, Germany) and showed in Table 1.

Table 1 Water contact angle of the modified and unmodified nanofiber membranes.

\begin{tabular}{|c|c|}
\hline Membrane & WCA $\left(^{\circ}\right)$ \\
\hline PA6 & 78.92 \\
\hline PVDF & 89.41 \\
\hline PAN & 80.72 \\
\hline PVDF-OH & 0 \\
\hline PAN-OH & 0 \\
\hline
\end{tabular}

A scanning electron microscope (SEM) is used to detect if any changes or damages on the membrane surface. Fiber diameters are measured using the ImageJ program. The oil-water emulsion was prepared as half-to-half oil and water in weight by adding $2 \%$ wt of triton X surfactant (Sigma). The oil/water/surfactant mixture was left to be mixed on the magnetic stirrer for $24 \mathrm{~h}$, speed @500 rpm. Amicon mini stirrer dead-end unit used for the filtration test. Flux $(f)\left(\mathrm{Lm}^{-2} \mathrm{~h}^{-1}\right)$ and permeability $(k)\left(\mathrm{Lm}^{-2} \mathrm{~h}^{-1}\right.$ bar $\left.^{-1}\right)$ were calculated according to Eq. (1) and (2).

$f=\frac{1}{A} \frac{d V}{d t}$ 
$k=\frac{f}{P}$

where

$V$ - the total volume of the permeate $(\mathrm{L})$

$A$ - the active membrane area (m2),

$t$ - the filtration time $(\mathrm{h})$,

$P$-the transmembrane pressure (bar).

\section{RESULTS AND DISCUSSION}

The surface of the membranes has been examined using SEM images after the lamination process (Figure 1). Lamination and modification do not damage the surface of nanofiber membranes.
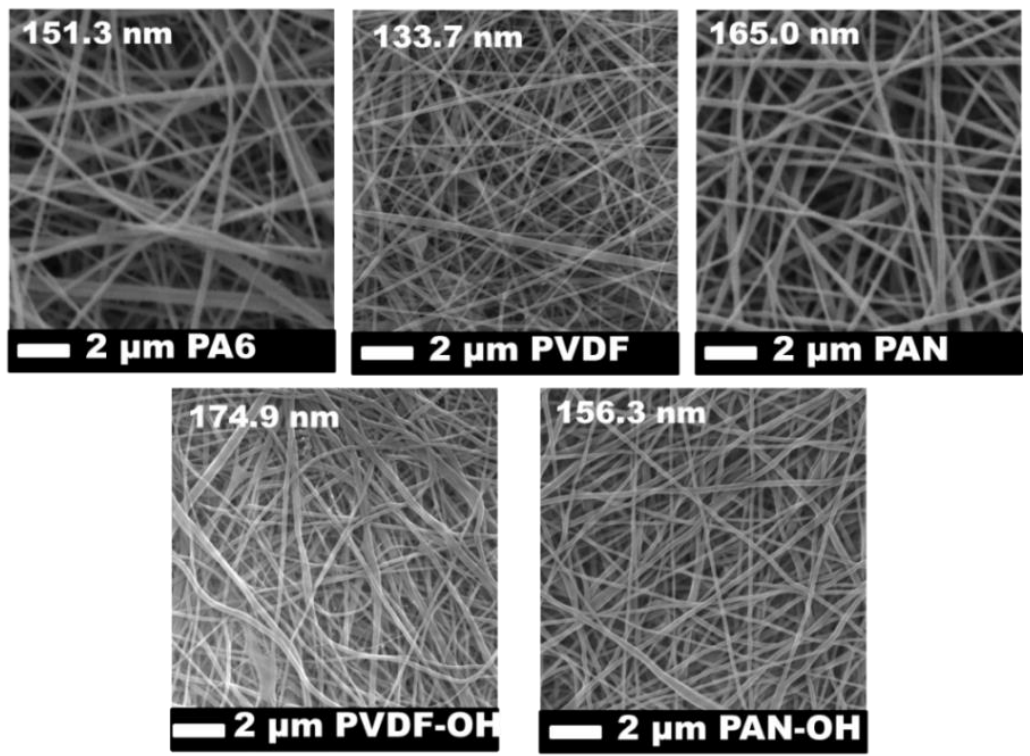

Figure 1 SEM images of the nanofiber membranes

The modified membranes submerged in distilled water which showed a highly hydrophilic structure. The reason is due to alkaline treatment which incorporates hydrophilic groups (such as hydroxyl or carboxyl) on PVDF and PAN membrane surface. It was found that the alkaline solution treated PAN membrane showed superhydrophilic/underwater superoleophobic characteristics due to $-\mathrm{CN}$ groups of PAN hydrolyzed to $\mathrm{COOH}$ groups [6,7]. Using alkaline treatment on the PVDF membrane caused dehydrofluorination, and hydroxyl groups formed on the surface $[8,9]$. The interaction between the alkaline solution and PVDF and PAN membrane has been given in Figure 2. From Table 1, it is visible that the modification process improved membrane hydrophilicity. Membrane hydrophilicity plays an important role in membrane flux, permeability, and selectivity. It was found that hydrophilic membranes reduce membrane fouling $[10,11]$.

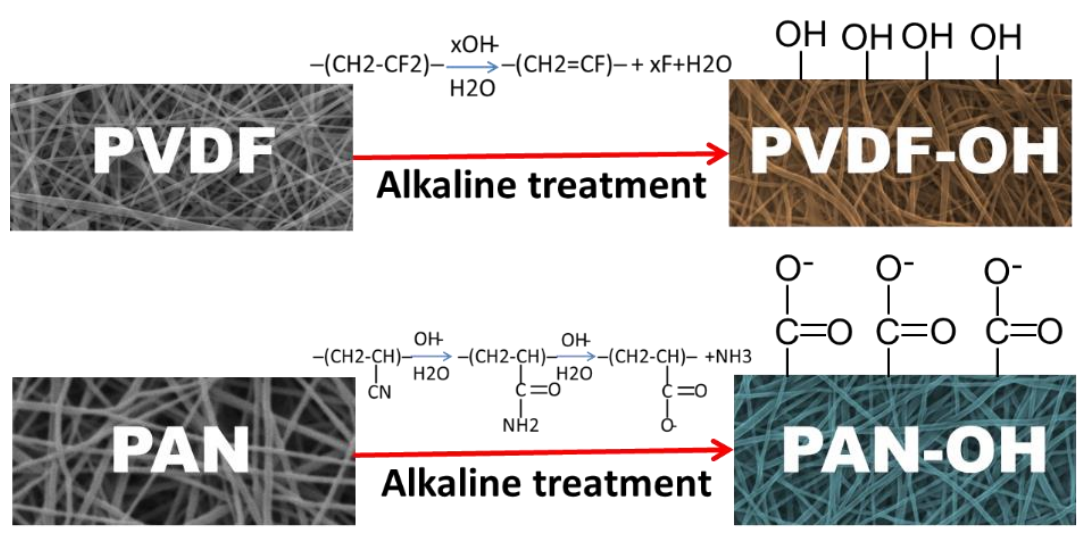

Figure 2 Surface modification of PVDF and PAN membranes 
Further experiments have been done to evaluate membrane permeability and selectivity for oil-water emulsion (Figure 3). For the filtration test, first distilled water, then emulsion was used. This step was followed 3 times. For each step, the same membrane has been used to observe membrane fouling. The applied pressure was almost zero ( 0.02 bar). All the membranes permeated water from the oil-water emulsion. The permeability of unmodified membranes decreased in each cycle due to membrane fouling. Oil droplets were trapped into membrane pores and caused membrane fouling. Increasing membrane wettability improves the attachment of water molecules to the membrane surface while repels the oil droplets and reduce their attachment to the membrane surface. As a result, membrane fouling resistance improves. Herein, after surface modification, both PAN and PVDF membranes showed remarkable membrane fouling resistance. The PVDF nanofiber membrane's permeability has not been changed in each cycle. On the other side, the permeability of the PAN membrane increased in each cycle. The reason could be due to the interaction of surfactant with $\mathrm{PAN}-\mathrm{OH}$ nanofiber membranes which improved membrane wetting in time.

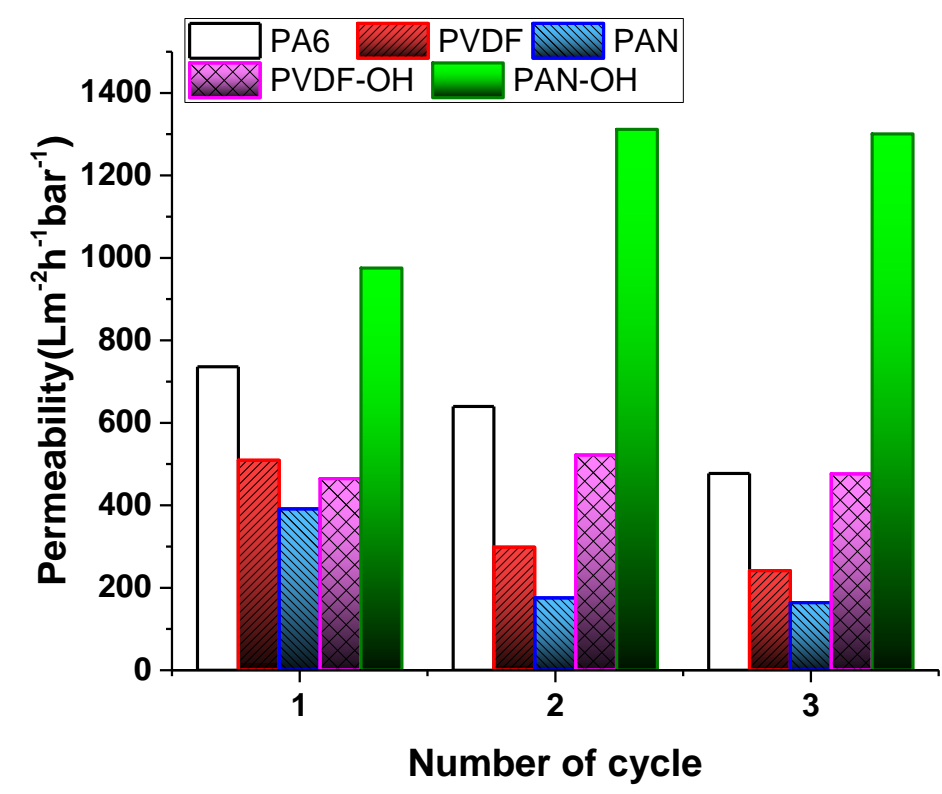

Figure 3 Permeability of modified and unmodified membranes

It was found that the surfactant showed to render the surface of the membranes more 'homogeneously' permeable, reduced flux decline, increased the flux, reduced the surface rugosity, and increased hydrophilicity $[12,13]$.

\section{CONCLUSION}

In this work, we have successfully prepared PVDF, PAN, and PA6 nanofiber membranes for the separation of oil-water emulsion. The surfaces of the PVDF and PAN membranes have been modified using the alkaline treatment to improve membrane hydrophilicity. The surface of the membranes did not damage after the lamination and modification process. Membrane modification reduced membrane fouling during filtration. Moreover, membrane water permeability was improved due to hydrophilic groups attached to the PVDF and PAN membranes. Overall, the super hydrophilic PVDF and PAN membranes with effective oil-water separation highlight the greatly promising potential for further applications in industrial wastewater treatment.

\section{ACKNOWLEDGEMENTS}

This work was supported by the Ministry of Education, Youth and Sports for Institutional support for Long-term Conceptual Development of a Research Organization of the Czech Republic, 2020. 


\section{REFERENCES}

[1] SINGH, V., PURKAIT, M.K., DAS, C. Cross-Flow Microfiltration of Industrial Oily Wastewater: Experimental and Theoretical Consideration. Separation Science and Technology. 2011, vol. 46, no. 8, pp. 1213-23.

[2] ZHAO, X., SU, Y., CHEN, W., PENG, J., JIANG, Z. Grafting perfluoroalkyl groups onto polyacrylonitrile membrane surface for improved fouling release property. Journal of Membrane Science. 2012, vol. 415-416, pp. 824-34.

[3] XU, Z., MIYAZAKI, K., HORI, T. Fabrication of polydopamine-coated superhydrophobic fabrics for oil/water separation and self-cleaning. Applied Surface Science. 2016, vol. 370, pp. 243-51.

[4] SADEGHI, I., AROUJALIAN, A., RAISI, A., DABIR, B., FATHIZADEH, M. Surface modification of polyethersulfone ultrafiltration membranes by corona air plasma for separation of oil/water emulsions. Journal of Membrane Science. 2013, vol. 430, pp. 24-36.

[5] TANG, H., HAO, L., CHEN, J., WANG, F., ZHANG, H., GUO, Y. Surface Modification to Fabricate Superhydrophobic and Superoleophilic Alumina Membranes for Oil/Water Separation. Energy Fuels. 2018, vol. 32, no. 3, pp. 3627-36.

[6] ZHANG, F., GAO, S., ZHU, Y., JIN, J. Alkaline-induced superhydrophilic/underwater superoleophobic polyacrylonitrile membranes with ultralow oil-adhesion for high-efficient oil/water separation. Journal of Membrane Science. 2016, vol. 513, pp. 67-73.

[7] HU, X.M., XIAO, C.F. Characterization and Hydrolization of PAN by Sodium Hydroxide. Advanced Materials Research. [online]. 2011 [viewed 2019 Jul 19]. Available from: https://www.scientific.net/AMR.332-334.1032.

[8] BOYRAZ, E., YALCINKAYA, F., HRUZA, J., MARYSKA, J. Surface-Modified Nanofibrous PVDF Membranes for Liquid Separation Technology. Materials. 2019, vol. 12, no. 17, p. 2702.

[9] MARCHAND BRYNAERT, J., JONGEN, N., DEWEZ, J.-L. Surface hydroxylation of poly(vinylidene fluoride) (PVDF) film. Journal of Polymer Science Part A: Polymer Chemistry. 1997, vol. 35, no. 7, pp. 1227-35.

[10] YALCINKAYA, F., SIEKIERKA, A., BRYJAK, M. Preparation of Fouling-Resistant Nanofibrous Composite Membranes for Separation of Oily Wastewater. Polymers (Basel). [online]. 2017 [viewed 2019 Jul 15], vol. 9, no. 12. Available from: https://www.ncbi.n/m.nih.gov/pmc/articles/PMC6418811/.

[11] YALCINKAYA, F., SIEKIERKA, A., BRYJAK, M. Surface modification of electrospun nanofibrous membranes for oily wastewater separation. RSC Advances. 2017, vol. 7, no. 89, pp. 56704-12.

[12] FANE, A.G., FELL, C.J.D., KIM, K.J. The effect of surfactant pretreatment on the ultrafiltration of proteins. Desalination. 1985, vol. 53, no. 1, pp. 37-55.

[13] KOLEY, D., BARD, A.J. Triton X-100 concentration effects on membrane permeability of a single HeLa cell by scanning electrochemical microscopy (SECM). PNAS. 2010, vol. 107, no. 39, pp. 16783-7. 